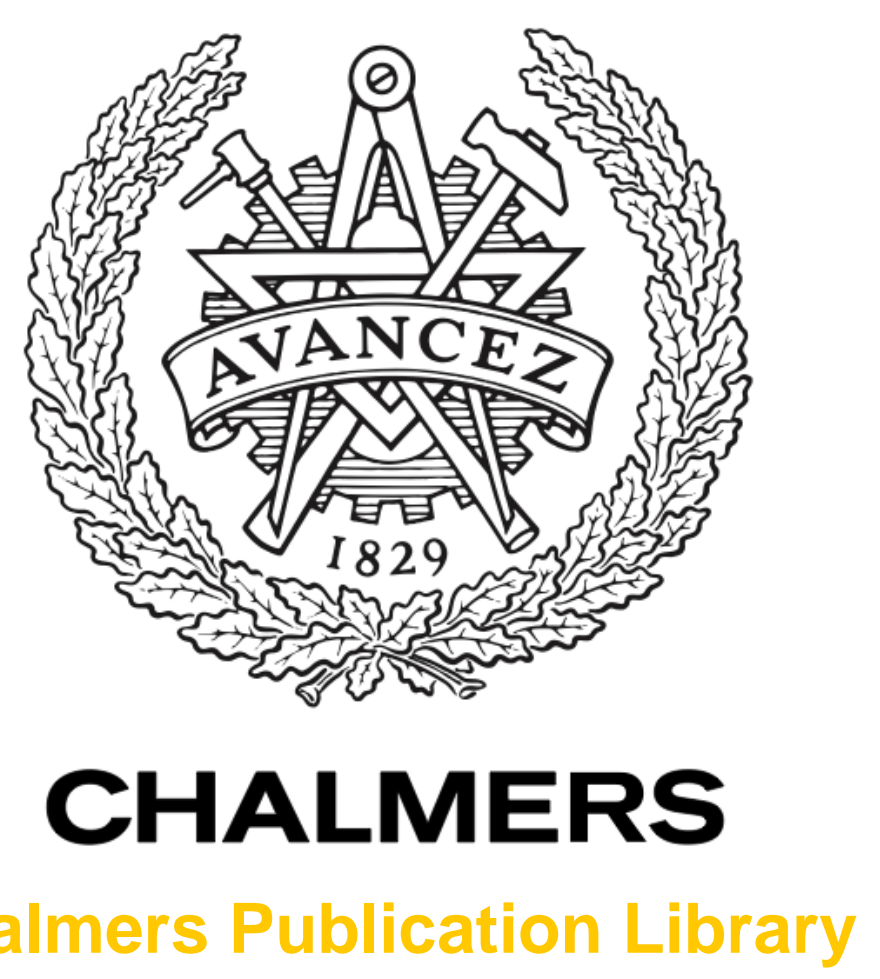

Chalmers Publication Library

\title{
Spectrum Superposition Based Chromatic Dispersion Estimation for Digital Coherent Receivers
}

This document has been downloaded from Chalmers Publication Library $(\mathrm{CPL})$. It is the author's version of a work that was accepted for publication in:

\section{European Conference on Optical Communications (ECOC 2014)}

Citation for the published paper:

Shuchang, Y. ; Eriksson, T. ; Songnian, F. (2014) "Spectrum Superposition Based Chromatic Dispersion Estimation for Digital Coherent Receivers". European Conference on Optical Communications (ECOC 2014) pp. P.3.17.

Downloaded from: http://publications.lib.chalmers.se/publication/207899

Notice: Changes introduced as a result of publishing processes such as copy-editing and formatting may not be reflected in this document. For a definitive version of this work, please refer to the published source. Please note that access to the published version might require a subscription. 


\title{
Spectrum Superposition Based Chromatic Dispersion Estimation for Digital Coherent Receivers
}

\author{
Shuchang Yao (1), Tobias A. Eriksson(2), Songnian Fu (1)*, Jianqiang Li (3), Pontus Johannisson(2), \\ Magnus Karlsson(2), Peter A. Andrekson(2), Perry Shum (1),(4), Deming Liu(1)
}

\begin{abstract}
(1)Next Generation Internet Access National Engineering Lab (NGIA), School of optical and electronic information, Huazhong University of Sci\&Tech (HUST), 1037 Luoyu Road, Wuhan, China 430074, *songnian@mail.hust.edu.cn (2)Photonics Lab, Department of Microtechnology and Nanoscience, Chalmers University of Technology, 41296, Sweden

(3) State Key Laboratory of Information Photonics and Optical Communications, Beijing University of Posts and Telecommunications, Beijing, 100876, China

(4)School of EEE, Nanyang Technological University, Singapore 637553
\end{abstract}

\begin{abstract}
We propose and experimentally demonstrate a fast blind CD estimation method based on signal spectrum superposition. With only 4096 samples, a maximum estimation error of $0.25 \%$ of the accumulated CD for $7 \times 112$ Gbps DP-QPSK WDM signal is verified.
\end{abstract}

\section{Introduction}

Fast chromatic dispersion (CD) estimation is a very important enabling technology in flexible and dynamic optical networking. By analyzing the impulse response of the adaptive CMA equalizer, one can quickly estimate $C D$ in a range of $0-1500 \mathrm{ps} / \mathrm{nm}$ depending on the number of taps ${ }^{1}$. Other blind $C D$ estimation methods like the maximum likelihood criterion method ${ }^{2}$, the best-match-search (BMS) estimation ${ }^{3}$, peak-toaverage-power ratio estimation ${ }^{4}$ and the delay-tap sampling (DTS) estimation ${ }^{5}$ usually estimate the accumulated $C D$ by scanning over a specific CD range. To guarantee the estimation accuracy, a large number of tentative compensation or tentative calculations of different cost functions are necessary for these schemes. The auto-correlation of signal power waveform (ACSPW) CD estimator was proposed for long-haul fiber link by directly searching the peak of the auto-correlation of the signal power ${ }^{6}$. About 8192 symbols were required for robust estimation of more than $10^{5} \mathrm{ps} / \mathrm{nm}$ of accumulated CD for 112 Gbps DPQPSK system ${ }^{6}$. Usually, the auto-correlation of the signal power was efficiently performed by employing the fast Fourier transform (FFT) ${ }^{6-7}$. Nevertheless, for the FFT calculation of the $2 \times 8192$ samples, it can be very difficult to realize in real digital coherent receivers because of its high implementation complexity.

In this paper, we propose a signal spectrum superposition based CD estimation method. Instead of calculating the autocorrelation of the signal power waveform as in [6], we superimpose the signal spectrum to estimate the $C D$ information. A higher peak-to-average-ratio (PAR) is observed since the noise is averaged after the spectrum superposition. Through simulations, we see that only 4096 samples can be used to estimate up to $10^{5} \mathrm{ps} / \mathrm{nm}$ of accumulated CD by our proposed method. Moreover, the proposed method is verified to be robust to amplified spontaneous emission (ASE) noise, phase noise, frequency offset and polarization mode dispersion (PMD) by a standard deviation within 20 $\mathrm{ps} / \mathrm{nm}$ in all simulation cases. We also experimentally verify our scheme by estimating the accumulated $C D$ for the $7 \times 112$ Gbps PM-QPSK WDM signals after 480 $\mathrm{km}-7200 \mathrm{~km}$ single mode fiber (SMF) transmission.

\section{Operation principle}

For the ease of discussion, the effect of PMD, phase noise and frequency offset is ignored first. Assume the modulated signal, with its spectrum denoted by $Q(n)$, is transmitted through a fiber link with a total length of $L$ and group velocity dispersion coefficient of $\beta_{2}$. At the receiver side, the FFT of the coherently received samples $\mathrm{S}(n)$ can be written as

$$
\begin{aligned}
S(n) & =Q(n) H(n)+G(n) \\
& =Q(n) \exp \left(2 j \pi^{2} \beta_{2} L\left(n R_{s} / N\right)^{2}\right)+G(n),
\end{aligned}
$$

where $H(n)$ is the frequency response of the CD, $G(n)$ is the noise spectrum, $N$ is the FFT block-size, $-N / 2 \leq n \leq N / 2-1$, and $R_{s}$ represents the sampling rate which is equal to twice the symbol rate.

We note that for $H(n)$, the quadratic phase term is difficult to detect. However this term can be transferred into a first-order amplitude term by introducing the superposition of $H(n)$ as

$$
\begin{aligned}
|P(n)|^{2} & =|H(n)+H(n+N / 2)|^{2} \\
& =2+2 \cos \left(2 \pi^{2} \beta_{2} L R_{s}^{2}(n / N+1 / 4)\right),
\end{aligned}
$$

where $P(n)$ is the superposed spectrum, $-N / 2 \leq n \leq-1$. Meanwhile, the first-order amplitude term can be easily 
detected by performing the inverse fast Fourier transform (IFFT) as

$$
\left|\operatorname{IFFT}\left(|P(n)|^{2}\right)\right|=2 \delta(\tau)+\delta\left(\tau+\tau_{0}\right)+\delta\left(\tau-\tau_{0}\right),
$$

where the peak located at $\tau_{0}=\pi \beta_{2} L R_{s}$ indicates an accumulated $C D$ as

$$
C D_{\text {est }}=-\frac{2 \pi c \beta_{2} L}{\lambda^{2}}=-\frac{2 c \tau_{0}}{\lambda^{2} R_{\mathrm{s}}} .
$$

The result in Eq. (2)-(4) suggest estimating the accumulated $C D$ by doing the superposition of received signal spectrum $\mathrm{S}(n)$. In order to maximize the peak under search, we apply a digital filter $F(n)$ with its tap weights satisfying

$$
\mathrm{Q}(n) F(n)=Q(n+N / 2) F(n+N / 2) .
$$

Then the CD can be estimated from

$$
R(\tau)=\left|\operatorname{IFFT}\left(|\mathrm{S}(n) \mathrm{F}(\mathrm{n})+\mathrm{S}(\mathrm{n}+\mathrm{N} / 2) \mathrm{F}(\mathrm{n}+\mathrm{N} / 2)|^{2}\right)\right| .
$$

Another benefit of the spectrum superposition is that the random noise $G(n)$ can be averaged. Thus a lower noise floor is achieved.

Through simulation, a typical plot of $R(\tau)$ for our proposed method applied for 112 Gbps NRZ DP-QPSK signal is shown in Fig. 1 (a)-(b). The digital filter in Eq. (5) is chosen as $F(n)=1+\cos (3 \pi n / N)$. The optical signal-to-noise ratio (OSNR) at $0.1 \mathrm{~nm}$ noise bandwidth is set to $20 \mathrm{~dB}$, and 4096 samples are used. Since our method exhibits a similar searching peak as the ACSPW method, we also plot the corresponding peaks for the ACSPW method in Fig. 1 (c)-(d) for a comparison. Clearly, with the same number of samples, our proposed method produces a higher peak amplitude and lower noise floor which results in a higher PAR. Thus, it can be more robust to environmental perturbations.
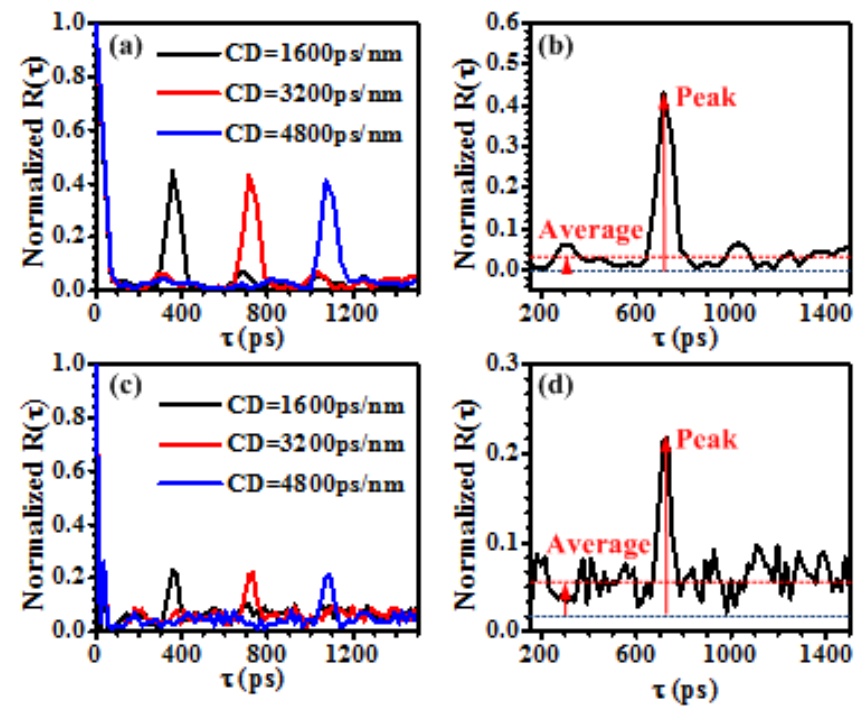

Fig. 1: CD estimation peaks for the 112 Gbps NRZ PM-QPSK signal with various accumulated CD using (a)-(b) our proposed method, (c)(d) ACSPW method.
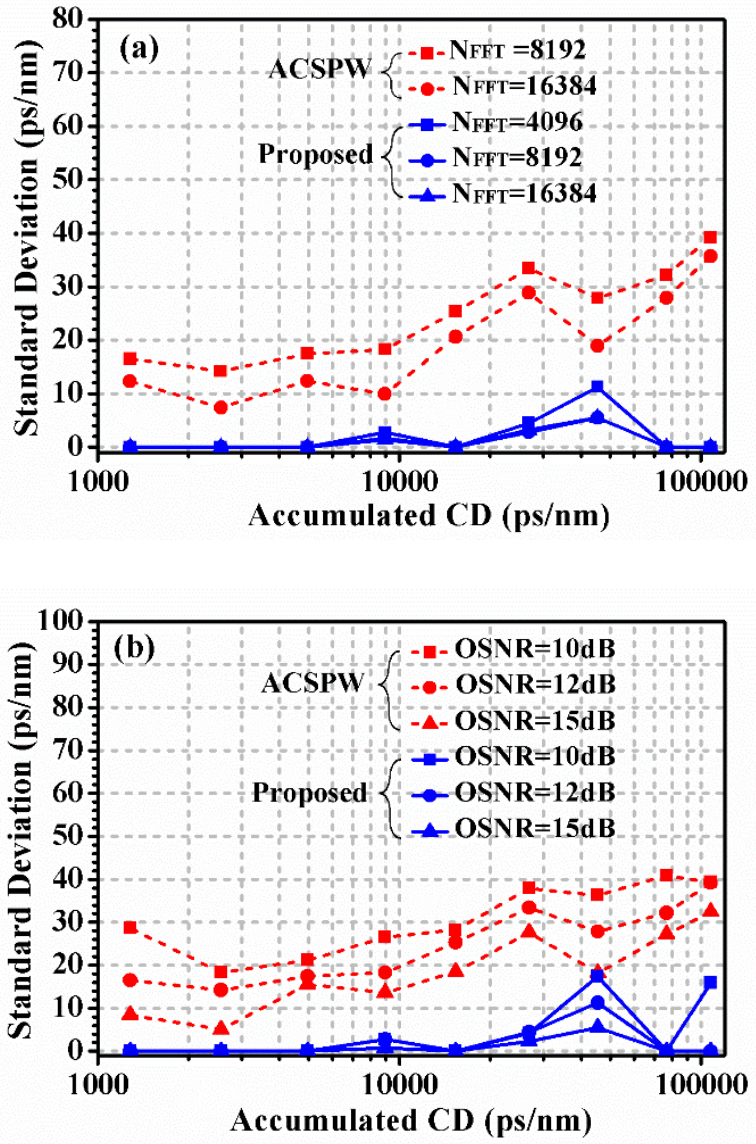

Fig. 2: (a) Standard deviation of $C D$ estimation error versus accumulated CD for 112 Gbps NRZ DP-QPSK systems, (b) standard deviation of $C D$ estimation error in the presence of different OSNR.

\section{Simulation and experimental results}

Using simulation in VPI Transmission Maker 9.0, investigations of the proposed $C D$ estimation method are carried out for the single carrier 112 Gbps NRZ DPQPSK system. Signals with a word length of $2^{17-1}$ are launched to the $80 \mathrm{~km}-6000 \mathrm{~km}$ SMF link. The CD coefficient of the SMF is $16 \mathrm{ps} / \mathrm{nm} / \mathrm{km}$, while the mean differential group delay (DGD) is varied from 0 ps-30 ps in all scenarios. At the coherent receiver side, a $5^{\text {th }}$ order Bessel electrical filter with $0.75 \times$ symbol rate bandwidth is applied before the received signals are sampled at twice the symbol rate. Both the signal source and the local oscillator (LO) have a linewidth of $1 \mathrm{kHz}-1 \mathrm{MHz}$ with their frequency offset randomly varying from $-2 \mathrm{GHz}$ to $2 \mathrm{GHz}$. The OSNR is set at 12 $\mathrm{dB}$ initially. The statistical results of $\mathrm{CD}$ estimation are shown in Fig. 2. At each accumulated CD value, 100 independent trials are conducted. We first compare the CD estimation performance of our proposed method with the ACSPW method. As shown in Fig. 2 (a), for both methods, the reduction of FFT block-size brings an increase of the standard deviation of the estimation error. However, the PAR is too low for the ACSPW method to operate properly with the FFT block-size 


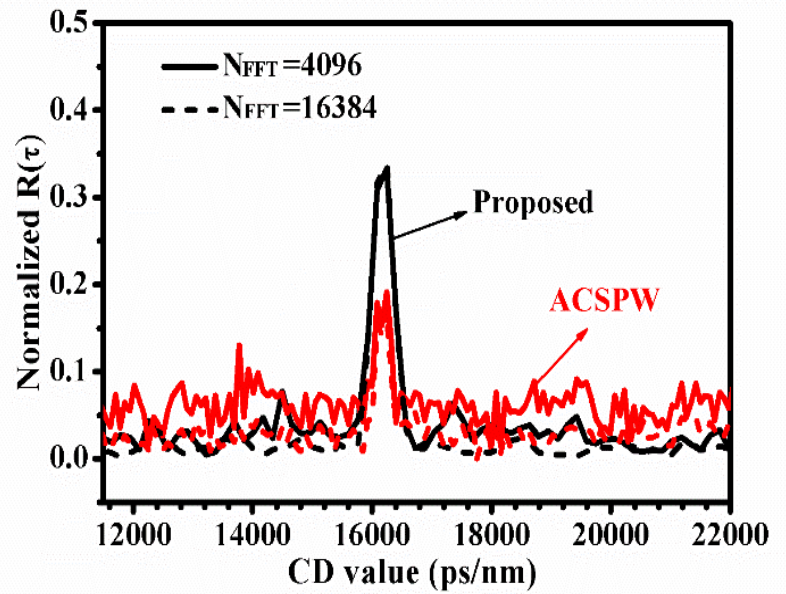

Fig. 3: Experimental CD estimation result for $7 \times 112$ Gbps NRZ DP-QPSK WDM after $960 \mathrm{~km}$ fiber link. Red plot for ACSPW method, and black for our proposed scheme.

(NFFT) of only 4096. On the other hand, due to the high PAR of our proposed method, the standard deviation of estimation error is much smaller than that of the ACSPW method. With a FFT block-size of 4096, the standard deviation for our method is lower than 20 $\mathrm{ps} / \mathrm{nm}$. We then investigate the estimation performance under different OSNRs, when the FFT block-size is fixed to 4096 for our proposed method and 8192 for the ACSPW method. As shown in Fig. 2 (b), compared to the ACSPW method, our proposed method is insensitive to the ASE noise with a smaller standard deviation, when the OSNR is varied from $10 \mathrm{~dB}$ to 15 $\mathrm{dB}$.

In the experiment, $7 \times 112$ Gbps NRZ DP-QPSK WDM signal with a channel spacing of $50 \mathrm{GHz}$ was transmitted over a recirculation loop of $\mathrm{k} \times 480 \mathrm{~km}$ SMF link, $k=1,2, \ldots, 15$. The launch power was $-6 \mathrm{dBm}$ per channel. A real-time oscilloscope with a sampling rate of $100 \mathrm{GSa} / \mathrm{s}$ was used to collect the received signals. The received signal was then resampled at 2 samples per symbol for the blind CD estimation. Fig. 3 shows the estimation peak using both ACSPW method and the proposed method after $960 \mathrm{~km}$ fiber link. The PAR of the estimation peak with the proposed method is much higher than that of the ACSPW method. Decreasing the FFT block-size leads to an increase of the noise floor. In fact, the estimation peak for ACSPW method is so small that it can be difficult to detect with a FFT block-size of 4096. Thus, a larger FFT block-size of 16384 must be used for the ACSPW method to improve its robustness against noise.

The statistical results of CD estimation is shown in Fig. 4. For each transmission distance, 1000 independent estimation is processed by using different received samples. In order to avoid significant estimation errors, the ACSPW has to be applied with 16384 samples while the proposed scheme uses only 4096 samples. Shown in Fig. 4, the maximum estimation error for the proposed method is no more than $0.25 \%$ of accumulated CD. The reason why the proposed method results in a slightly larger error range than the ACSPW method is that after spectrum superposition, the time resolution for our method is twice that of ACSPW.

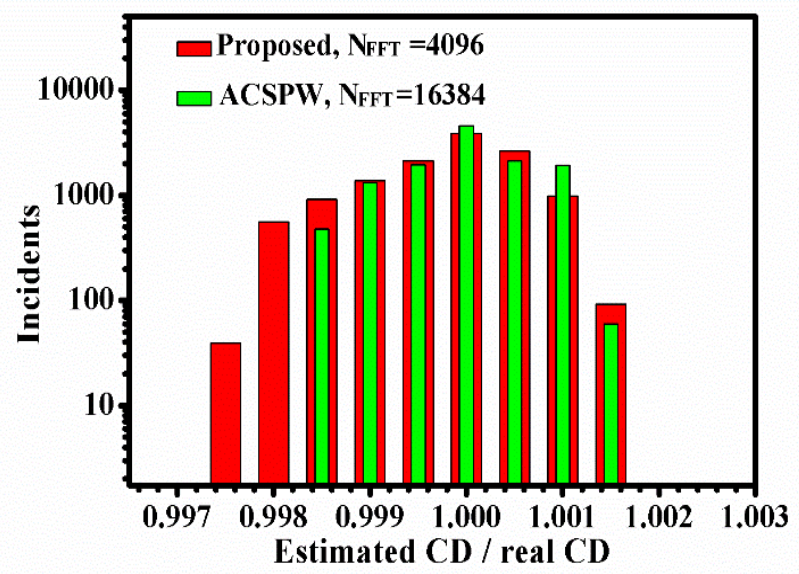

Fig. 4: Experimental results for $7 \times 112$ Gbps NRZ DP-QPSK WDM after $\mathrm{k} \times 480 \mathrm{~km}$ fiber loop link.

\section{Conclusion}

A fast blind CD estimation method is proposed and experimentally demonstrated for $7 \times 112 \mathrm{Gbps}$ DPQPSK WDM signal with a maximum estimation error of $0.25 \%$ of the accumulated CD after $480 \mathrm{~km}-7200 \mathrm{~km}$ optical fiber transmission. With a required FFT blocksize of 4096, it can be applicable for coherent receiver implementation.

\section{Acknowledgements}

This work was supported by the National Basic Research Program of China (973 Program: 2010CB328302), the 863 High Technology Plan (2012AA011301), and the National Natural Science Foundation of China (61275069).

\section{References}

[1] F. N. Hauske et al., J. Lightwave Technol, 27, 16, p.3623, (2009)

[2] H. Wymeersch et al., J. Lightwave Technol., 30, 18, p.2976, (2012)

[3] R. Soriano et al., J. Lightwave Technol., 29, 11, p.1627, (2011)

[4] C. Xie et al., Photonics Technol. Letters, 25, 10, p.985, (2013).

[5] D. Wang et al., Photonics Technol. Letters, 23, 14, p.1016, (2011).

[6] Q. Sui et al., J. Lightwave Technol., 31, 2, p.306, (2013).

[7] F. Pereira et al., Photonics Journal., 5, 4, p.1943, (2013). 